\title{
Ultrasonic techniques for assessment of temperature and thermal damage
}

\section{Emad Ebbini}

Emad S. Ebbini, "Ultrasonic techniques for assessment of temperature and thermal damage," Proc. SPIE 10297, Matching the Energy Source to the Clinical Need: A Critical Review, 102970 (24 January 2000); doi: 10.1117/12.375219

SPIE. Event: Digital Optical Computing, 1990, Los Angeles, United States 


\title{
Ultrasonic techniques for assessment of temperature and thermal damage
}

\author{
Emad S. Ebbini \\ Department of Electrical and Computer Engineering \\ University of Minnesota, Minneapolis, MN 55455, USA
}

\begin{abstract}
The use of diagnostic ultrasound as a tool for noninvasive temperature feedback, image guidance, and damage assessment is described. The physical principles allowing for such applications are discussed along with the underlying scattering models. It is shown that temperature changes on the order of $0.1^{\circ} \mathrm{C}$ can be detected with a spatial resolution on the order of $1 \mathrm{~mm}$. It is further shown that temperature variations can be tracked up to nearly $20^{\circ} \mathrm{C}$ from baseline for relatively long durations. In addition to temperature feedback, the potential for using ultrasound for damage assessment is discussed. Finally, the latest efforts on new self-guided ultrasonic phased array systems for imaging and therapy are discussed. The paper is concluded with a discussion of the future directions that will ultimately define the role of diagnostic and therapeutic ultrasound in the general area of image-guided surgery.
\end{abstract}

Keywords: Noninvasive surgery, image guidance, treatment monitoring, ultrasonic imaging, phased arrays, autoregressive modeling.

\section{INTRODUCTION}

Significant advances in the general area of minimally invasive and noninvasive surgery have been made in the last few years. This can be attributed to advances in key enabling technologies in medical imaging, computers, and instrumentation. Thermal ablative and coagulative therapies utilizing various sources of heat have received significant attention due to the inherent safety and absence of harmful side effects when the energy source is controlled properly. Several research groups and industrial entities are developing image-guided minimally invasive or noninvasive techniques for thermal surgery with applications ranging from cardiac ablation to cancer treatments.

In terms of energy sources, there are many forms of applicators with various degrees of sophistication and emplying a variety of modalities for tissue coagulation. Most of the available applicators have been optimized for specific tissue targets and desired coagulated volume based on reasonable, but imperfect, theoretical models. Multi-element applicators are becoming common place today and offer

Other author information: E-mail: emad@ece.umn.edu

Matching the Energy Source to the Clinical Need: A Critical Review, edited by Thomas P. Ryan,

Proc. of SPIE Vol. 10297 (Vol. CR75), 102970I · (c) (2000) 2017 SPIE

CCC code: $0277-786 X / 17 / \$ 18 \cdot$ doi: $10.1117 / 12.375219$ 
improved control over the coagulated volume. Without adequate temperature feedback, however, these additional degrees of freedom are rendered useless. Therefore, this brings us to the second major development area for the future of minimally invasive surgery. In particular, the development of noninvasive or minimally invasive methods for guidance of the energy source and monitoring the tissue response during thermal surgery.

\subsection{Image Guidance}

Image guidance for noninvasive thermal therapy is now a reality. This may be based on well established imaging modalities like MRI, ${ }^{15} \mathrm{CT},{ }^{19}$ or ultrasound. ${ }^{16,18}$ Other imaging modalities are also being developed and may become available in the foeseeable future. ${ }^{22}$ The main component of an image guidance system for thermal surgery appear to be the temperature imaging which has received significant attention during the last 10 - 15 years. Several modalities are being used for noninvasive temperature estimation such as $\mathrm{MR},{ }^{24-26} \mathrm{CT},{ }^{19}$ ultrasound. ${ }^{28,1,17,21,27,11}$ The specific imaging modality used for image guidance will depend on many factors such as doctor preference, target type and site, economics, etc. Nevertheless, some form of image guidance will definitely be available in the operating room in the near fututre.

\subsection{Temperature Imaging}

Among other forms of feedback, feedback information on the temperature evolution of the region being treated is needed. ${ }^{15,18} \mathrm{MRI}^{25,26} \mathrm{CT}^{24,19}$ impedance tomography, ${ }^{22}$ microwave radiation ${ }^{23}$ and ultrasound ${ }^{28,27,20,1,21,18,11}$ imaging have been studied for this purpose. Each of these modalities has its particular advantages and shortcomings. In this paper, we focus on the use of pulse-echo ultrasound in noninvasive temperature estimation. While the other methods, especially MRI, offer important advantages as means of noninvasive temperature estimation, ultrasound is very attractive due to several key advantages:

1. Real-time $2 \mathrm{D}$ temperature estimation; data can be acquired and processed at 8 frames a second and even higher.

2. High spatial resolution; currently the resolution of temperature estimation with ultrasound is on the order of $1 \mathrm{~mm}$.

3. High sensitivity; changes of tissue temperature on the order of $0.1^{\circ} \mathrm{C}$ can be detected easily with high spatial resolution.

4. Portability and compatibility with most heating equipment; for instance, we were able to use a commercially available B-scan system in imaging while high $\mathrm{RF}$ energy was being used with minimal coupling of the RF to the ultrasound image. 
Of course, temperature imaging with pulse-echo ultrasound has its limitations which are discussed below. More research is needed to overcome these limitations to allow for practical use of this temperature imaging method in clinical situations or in in-vivo animal testing. Some of these limitations are discussed in detail in earlier publications. ${ }^{12,13,11}$ They are enumerated in the discussion section below with appropriate citation.

\subsection{Image-Based Methods for Damage Assessment}

Based on the current state of the art, MRI and CT are expected to offer superior performance when it comes to damage assessment. However, these may be very expensive and complex. Ultrasound offers several key advantages for image guidance such as portability, compatibility, real-time operation, ease of use, etc. Unfortunately, however, gray-scale ultrasonic imaging does not offer quantitative measurements that relate to the state of the tissue. Therefore, ultrasound has not been considered a serious tool for noninvasive damage assessment of treated tissue. This view of ultrasonic imaging misses the fact that ultrasound backscattered pulses are a result of interaction between the basic ultrasound wavelet with the microstructure of the tissue. Therefore, with appropriate signal processing, ultrasound sholud provide some useful parameters that correlate with tissue state.

\subsection{Self-Guided Systems for Noninvasive Surgery}

Another area unique to ultrasound that could revolutionize the field of image guided surgery is the development of a new generation of dual-mode high-power phased array systems capable of both imaging and therapy. ${ }^{14}$ These piezocomposite transducers can produce focal intensity levels needed for ablative and coagulative theramal surgery with high precision. Furthermore, the operating bandwidth of such transducers allows for imaging the treatment region with adequate image quality to delineate important landmarks within and around the target volume. With these capabilities, it is possible to operate these arrays in a self-guided mode whereby the array's imaging capabilities are utilized to improve the quality of the therapeutic foci, guide the therapeutic foci to the (possibly moving) target, and even perform temperature imaging around the therapeutic foci.

\subsection{Organization and Scope of Paper}

The rest of the paper is organized as follows: Section 2 describes the theoretical bases, formulation, and the implementation of noninvasive two-dimensional methods for temperature estimation with pulse-echo ultrasound. Section 3 addresses the use of diagnostic ultrasound for damage assessment and characterization of thermal lesions. In Section 4 we describe a phased array system capable of performing both imaging and therapy and, therefore, offers the potential for self-guided noninvasive method for noninvasive surgery. Section 5 offers a discussion on the current status and future directions in the development of ultrasonic methods for noninvasive surgery. In Section 6 we offer some concluding remarks. 
The reader will undoubtedly realize that much of the material included in this paper is based largely on the author's work in this area for the last few years. This was done primarily for convenience given some time constraints. It should not be interpreted as a judgement on the author's part as to the worthiness of other related work in this area. However, we do stress the fact that the material included herein is represensative of the state of the art in the three major areas identified in this paper. We hope that the citations given will give the reader a more complete perspective of the scope of ongoing research in this area.

\section{TEMPERATURE IMAGING WITH PULSE-ECHO ULTRASOUND}

The temperature dependence of some of the acoustic propagation parameters, especially the speed of sound, has long been recognized and reasonably well understood. $^{8,9}$ However, early attempts at imaging temperature noninvasively were primarily based on transmission mode ${ }^{9}$ or tomographic reconstruction techniques. ${ }^{8}$ Unfortunately, these techniques have many limitations that hampered any significant progress in this area beyond simple laboratory demonstrations. Recently, however, several groups have proposed new methods for temperature estimation using pulse-echo ultrasound using spectral methods ${ }^{20,27}$ and echo shift methods. ${ }^{1,21,11}$

\subsection{Theoretical Formulation}

\subsubsection{Model}

The temperature-change estimation method described in this paper is based on the thermal dependence of the ultrasound echo that accounts for two different physical phenomena: (1) local change in speed of sound due to changes in temperature and (2) thermal expansion of the propagating medium. The former produces an apparent shift in scatterer location and the latter leads to a physical shift. Along an A-line, however, the two effects lead to echo time-shifts that can be estimated and are shown to be related to local change in temperature in the medium. In many situations of pratical interest, these effects are typically small, so that a linearized approach can be used in the analysis that follows. It should be mentioned, however, that for large temperature rise and/or large size of the heated region, the linearized analysis breaks down. Different formulation is needed to handle these cases. This formulation will be introduced in a future report.

The baseline temperature in the sample (tissue) medium is assumed to be constant and equal to $\theta_{0}$ at the initial time $T_{0}$. We start by considering the time delay of the echo from a scatterer at axial depth $z$ as being exclusively a function of the temperature-dependent speed of sound in the propagating medium:

$$
t_{c}(z)=2 \int_{0}^{z} \frac{d \xi}{c(\xi, \theta(\xi))}
$$

where $\theta(\xi)=\theta_{0}+\delta \theta(\xi)$ is the temperature at depth $\xi, c(\xi, \theta(\xi))$ represents the speed of sound at depth $\xi$ and temperature $\theta(\xi)$, and the subscript $c$ indicates 
464 / Critical Reviews Vol. CR75

that only the thermal dependence of speed of sound has been considered. In order to account for thermal expansion in the propagating medium we substitute $d \xi$ by $(1+\alpha(\xi) \delta \theta(\xi)) d \xi$ in Eq. (1), where $\alpha(\xi)$ is the linear coefficient of thermal expansion of the medium at axial depth $\xi$. We get:

$$
t(z)=2 \int_{0}^{z} \frac{1+\alpha(\xi) \delta \theta(\xi)}{c(\xi, \theta(\xi))} d \xi
$$

At the initial time $T_{0}=0$, the echo time delay is $t_{0}(z)=2 \int_{0}^{z} \frac{1}{c\left(\xi, \theta_{0}\right)} d \xi$. Once the temperature in the medium changes, a time-shift will be observed on the echo from scatterer at axial depth $z$ :

$$
\begin{aligned}
\delta t(z) & =t(z)-t_{0}(z) \\
& =2 \int_{0}^{z}\left[\frac{1+\alpha(\xi) \delta \theta(\xi)}{c(\xi, \theta(\xi))}-\frac{1}{c\left(\xi, \theta_{0}\right)}\right] d \xi .
\end{aligned}
$$

Differentiating Eq. (3) with respect to the scatterer location $z$, we get:

$$
\frac{\partial}{\partial z}(\delta t(z))=2\left[\frac{1+\alpha(z) \delta \theta(z)}{c(z, \theta(z))}-\frac{1}{c\left(z, \theta_{0}\right)}\right] .
$$

The thermal dependence of the speed of sound in tissue can be assumed to be approximately linear in the temperature range of interest ${ }^{9}$ :

$$
c(z, \theta(z))=c_{0}(z)(1+\beta(z) \delta \theta(z)),
$$

where $\beta(z)=\left.\frac{\partial c(z, \theta)}{\partial \theta}\right|_{\theta=\theta_{0}}$, and $c_{0}(z)=c\left(z, \theta_{0}\right)$. From Eqs. (4), (5), and using the fact that $|\beta(z) \delta \theta(z)| \ll 1$, solving for $\delta \theta(z)=\left(\theta(z)-\theta_{0}\right)$, we get:

$$
\delta \theta(z)=\frac{c_{0}(z)}{2}\left(\frac{1}{\alpha(z)-\beta(z)}\right) \cdot \frac{\partial}{\partial z}(\delta t(z)) .
$$

The term $k(z)=\frac{1}{\alpha(z)-\beta(z)}$ is a medium (material) dependent parameter. For a homogeneous medium this parameter can be experimentally determined. For the remainder of this paper we assume $k(z)$ and $c_{0}(z)$ to be invartiant with respect to axial depth $z$, i.e., we assume $k(z)=k$ and $c_{0}(z)=c_{0}$. In practice, these parameters should be allowed to vary in space and as a function with temperature. For the purposes of this paper, however, we will limit our attention to homogeneous media and small changes in temperature so that these assumptions are approximately valid. It should be mentioned that the linearized approach is valid for temperature changes on the order of $10-15^{\circ}$, even in inhomogeneous media. Therefore, the temperature estimation algorithm described below will be of practical for temperature estimation in hyperthermia and, more importantly, for image guidance of thermal therapy devices. 
Matching the Energy Source to the Clinical Need / 465

\subsection{Characterization}

$\mathrm{In}^{30,11}$ we have presented experimental data comparing noninvasive temperature estimation data from a tissue mimicking phantom with thermocouple measurements. The results of this test can be summarized as follows:

1. Sensitivity: Temperature changes on the order of $0.1-0.2{ }^{\circ} \mathrm{C}$ were estimated with sufficient signal to noise.

2. Spatial Resolution: Two dimensional temperature estimates of highly focused heating patterns were obtained. Frames with $\Delta T$ of 0.25 seconds were used to obtain 2D temperature maps of a single focus and extended heating patterns. Theoretically, the temperature rise should resemble the intensity profile of the heating pattern (this is the initial heating rate technique). Indeed, the temperature maps were almost identical to the intensity profiles as predicted by the theory. In this case the algorithm described in ${ }^{11}$ was used without filtering. It can be concluded that the temperature estimation algorithm has the same spatial resolution of the ultrasound imaging system used.

3. Temperature Artifacts: The heated region creates an aberrator (or lens) in the tissue sample. This aberrator causes the imaging beams to deflect when traversing the heated region, especially near temperature gradients. We have named this phenomenon the thermoacoustic lens effect. This lens effect produces ripple in the temperature map in the shadow of the heated region (when seen from the imaging array side). We have shown that filtering significantly reduces this ripple. However, this is achieved at the expense of spatial resolution.

\subsection{Applications}

In this section, we describe three applications of the temperature estimation algorithm as it stands at this point. The limitations noted below not withstanding, these applications demonstate the enormous potential and some of the distinct advantages of using ultrasound for noninvasive temperature feedback.

\subsubsection{Image Guidance for Noninvasive Surgery}

Figure 1 shows an experimental setup used in image guidance based on the temperature imaging method described in this paper. In this experiment, a thick piece of bovine muscle was subject to a sequence of heating patterns due to the therapeutic array. Eight 2-second duration foci were used for simulating a coagulative thermal necrosis in tissue. The individual foci were spaced by two $\mathrm{mm}$ and deivered to the target in sequence with the foci approaching the imaging transducer. Ample wait time between shots was given to allow temperature to return to baseline before the next shot is delivered. Upon completion of the experiment, the tissue sample was cut and it was confirmed that tissue destruction (as indicated by visible change of color) at the desired location. In order to demonstrate the capability of the temperature estimation algorithm for guiding the therapy, the sequence of 
466 / Critical Reviews Vol. CR75

shots were initially delivered at low power (causing only $2^{\circ} \mathrm{C}$ rise at the focal point. That is, the therapy array was operated well below the threshold for tissue damage and was run in a sequence that resembles the planned therapy session. While this was being done, temperature images were collected and processed to determine if the locations and quality of the foci are consistent with the treatment objectives. This allows for characterizing the quality of the heating foci in-situ at subthreshold (nondestructive) levels just before the theraputic pulses are to be delivered. Figure 2 shows the spatio-temporal temperature distribution along one image line going through all eight heating spots. The grayscale temperature image is obtained from an M-mode image consisting of $128 \mathrm{~A}$-lines. For each shot, $16 \mathrm{~A}$-lines were acquired and processed for temperature estimation, 2 before, 4 during, 5 after (fast rate), and 5 after (slow rate). Please note that the time axis is simply labeled A-line Number. For the temperature estimation experiment shown, each 16 lines represent 1 minute, but the $\mathrm{A}$-lines were not acquired uniformly in time. Figure 2

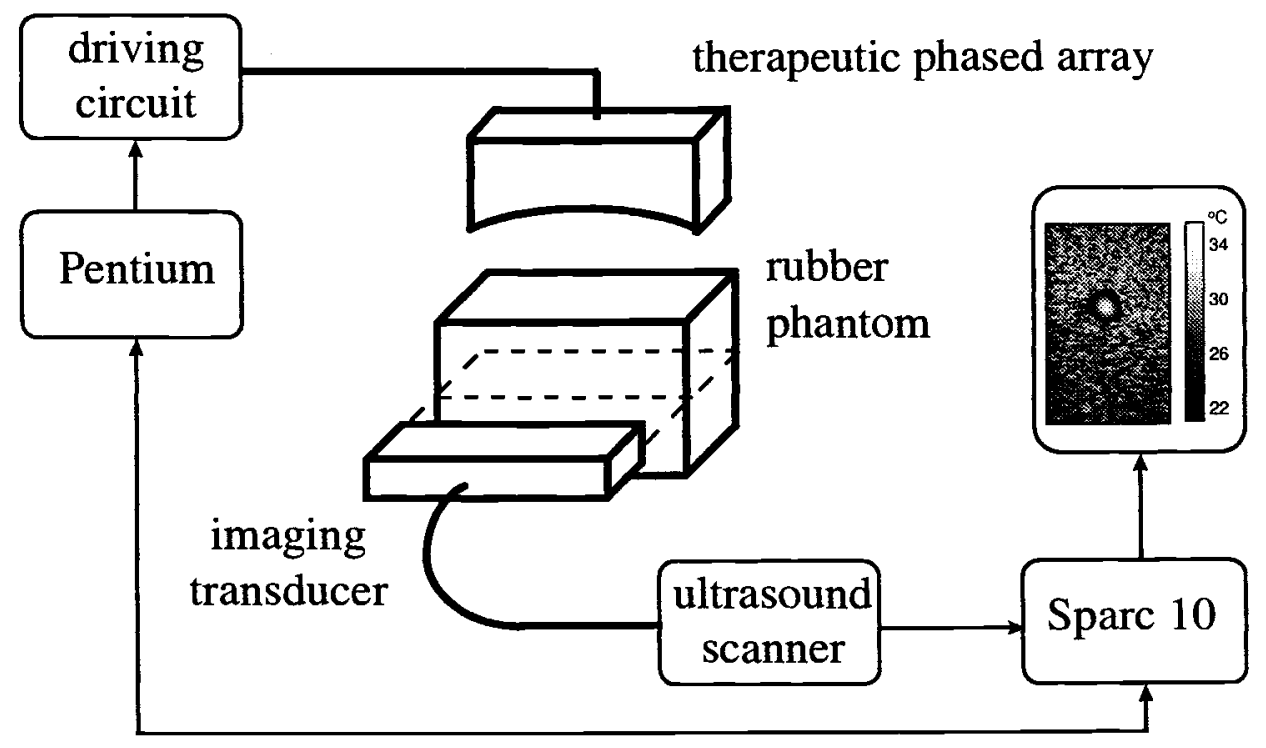

Figure 1. Experimental setup used in the image guidance experiment.

shows clearly that the temperature estimation algorithm has the spatial contrast and sensitivity needed to characterize the quality of the therapeutic transducer. The peaks of the temperature rise profiles are spaced by $2 \mathrm{~mm}$ as planned. Also, the extent of each heated region is between $3-5 \mathrm{~mm}$ axially which also corresponds to the expected temperature profile of a $2 \mathrm{~mm}$ wide beam heating for two seconds. While the image clearly demonstrate the potential of the temperature estimation algorithm, it should be noted that some level of ripple artifact exists. However, this ripple is not a limiting factor in this case. It should also be emphasized that the 


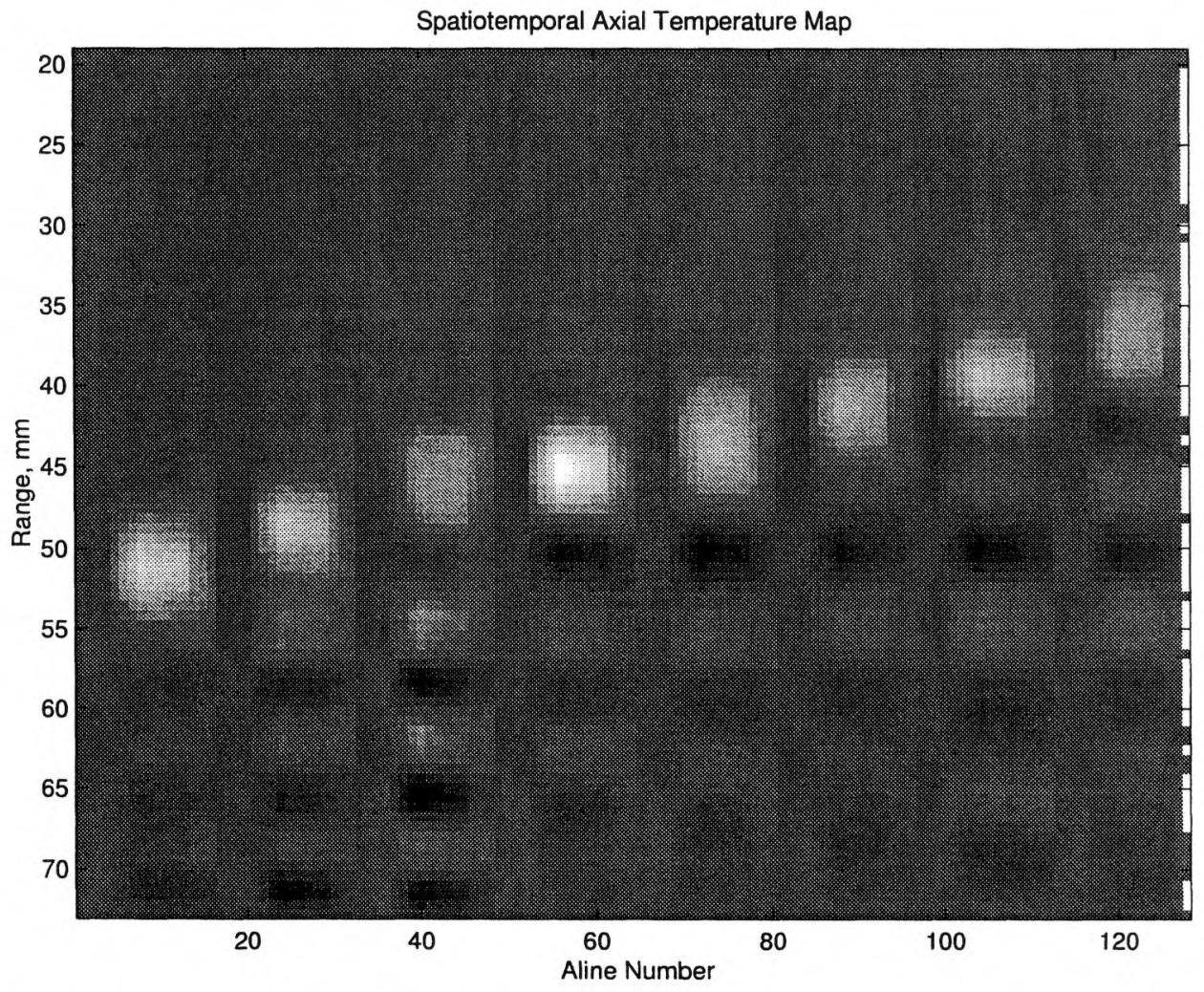

Figure 2. A spatio-temporal map of tissue temperatures along the axis of the imaging transducer.

maximum temperature rise needed to produce this result is approximately $2{ }^{\circ} \mathrm{C}$. This is a major advantage for ultrasound in that the quality assurance of focusing and energy deposition can be performed energy levels well below the therapeutic thresholds. Furthermore, since these images can be acquired and processed within a fraction of a second, the initial heating rate can be evaluated two dimensionally. Therefore, it is possible to visualize the specific absorption rate (SAR) of the energy source in situ. These are perhaps the major strengths of using ultrasound in image guidance of energy sources for noninvasive or minimally invasive thermal surgery.

\section{PULSE-ECHO IMAGING METHODS FOR DAMAGE ASSESSMENT}

At present, the issue of image-based damage assessment remains largely unsolved. To the best of our knowledge, no research group has made significant progress on this issue with any imaging modality, including MRI and CT. These modalities 
hold the most promise due to their quantitative nature. Ultrasound, on the other hand, suffers from some significant shortcomings due to nonquantitative nature of gray scale imaging and lack of 3D imaging in most clinical scanners. Nonetheless, ultrasound continues to be attractive due to its real-time imaging capability and high flexibility. In addition, with appropriate signal processing, quantitative tissue parameters can be extracted from pulse-echo data. While these tissue chacterization techniques have not been widely successful in identifying and classifying tissue pathology in normal clinical scenarios, it is expected that they will have a role to play in chacterization of thermal lesions. This is due to the drastic change of some of the acoustic properties within the ablated or coagulated volume, e.g., attenuation.

Imaging of attenuation in reflection mode has been investigated by many investigators for diagnostic purposes. One of the most successful algorithms for estimation of this parameter is based on low-order autoregressive (AR) modeling of backscattered ultrasonic signals. In what follows, we describe the use of this technique in an in-vitro experiment. While the results shown are encouraging, they are very preliminary and should be interpreted as an indicator of potential rather than true performance. Much more work needs to be done in this area for real prgress to be made.

\subsection{Theoretical Formulation}

\subsubsection{Backscattered Signal Model}

A widely accepted model for ultrasound backscattered signal from tissue media expresses the received signal as a superposition of reflections from $N$ scatterers within the resolution cell of the transducer:

$$
x(n)=\sum_{i=1}^{N} A_{i} p_{z}\left(t-\tau_{i}\right),
$$

where $A_{i}$ is the strength of the $i$ th scatterer assumed to be independent of frequency, $p_{z}(t)$ is the basic ultrasound wavelet emitted from the transducer with $z$ (range) dependence to account for attenuation, diffraction, etc., and $\tau_{i}$ is the two-way propagation delay to the $i$ th scatterer $\left(\tau_{i}=2 \frac{z_{i}}{c}\right)$. A simplified version of the spectrum of the bacscattered signal can be given by:

$$
X(f)=P(f) \sum_{i=1}^{N} A_{i} e^{-\frac{\alpha(f) c \tau_{i}}{8.68}} e^{-j 2 \pi f \tau_{i}}
$$

where we have explicitly accounted for the attenuation ( $\alpha$ is the attenuation coefficient in $\mathrm{dB} / \mathrm{cm}$ ).

\subsubsection{Autoregressive Modeling}

Autoregressive (AR) modeling of ultrasound backscattered data has proved to be extremely beneficial in many applications, e.g., Doppler, mean scatterer spacing 
estimation, attenuation measurements, etc. The $p$ th order AR model for the received signal given in Equation 7 is given by:

$$
x(t)=-\sum_{i=1}^{p} a_{i} x(n-i)+\epsilon(n),
$$

where $x(n)$ is the sampled version of $x(t)$ at $t=n T, a_{i}$ are the AR filter coefficients to be determined from the time series, and $\epsilon(n)$ is white Gaussian noise sequence with zero mean and variance $\sigma_{\epsilon}^{2}$.

A typical use of the AR model calls for a model order $p$ such that $\epsilon$ is truly a white noise sequence. However, for many applications, a low-order AR model is preferred for computational simplicity and robustness. Furthermore, for values of $p$ equal to 2 and 3 (AR2 and AR3), the coefiicients of the AR model take on physical significance with intuitive appeal. Regardless, once the model order has been determined the filter coefficients can be determined by many techniques based on second oder or higher order statistics. Furthermore, algorithms for determining the optimal filter coefficients have been developed for cases when the underlying model is stationary or nonstationary. The interested reader is referred to several textbooks on the subject.

\subsubsection{AR Parameter Estimation}

In an AR2 model, the coefficients $a_{1}$ and $a_{2}$ are direcly related to the reflection coefficients as well as the center frequency of the spectrum of the backscatter. The latter is an important parameter in determining the attenuation coefficients. The attenuation coefficient is known to change dramatically when the tissue is subjected to coagulative necrosis. ${ }^{29}$ Assuming that $\alpha$ is a linear function of frequency, $\alpha=$ $\alpha_{o} f$, then it can be shown that

$$
\alpha_{o}=\frac{-4.34}{c \sigma_{s}^{2}} \frac{d f_{o}}{d z},
$$

where $\sigma_{s}$ is related to the transducer bandwidth and $f_{o}$ is the center frequency of the backscattered signal. It can also be shown that

$$
a_{1}=-2 \cos \left(2 \pi \frac{f_{o}}{f_{s}}-\frac{8 \pi \sigma_{s}^{2}}{4.34 f_{s}} \alpha_{o}\right),
$$

where $f_{s}$ is the sampling frequency.

\subsection{Experimental Results}

The system shown in Figure 1 was used for producing a $1 \mathrm{~cm}^{3}$ lesion in an in-vitro bovine muscle. The lesion was formed by electronically scanning the therapy transducer to produce 10 contiguous HIFU spots in one plane. The therapy array was mechanically scanned to produce a three dimensional lesion in 10 plane. Ultrasound $\mathrm{B}$-scan imaages where acquired before and after the lesion formation. Examples of 
these B-scan gray scale images are shown in Figure 3. The lesion boundaries are shown by the box in Figure 3b). However, examining the B-scan image does not reveal any useful information about the lesion. This result is typical and it is the reason behind the perception that B-scan ultrasonic imaging does not provide quantitative information that can be utilized for quantitative analysis of tissue damage due to coagulative necrosis.

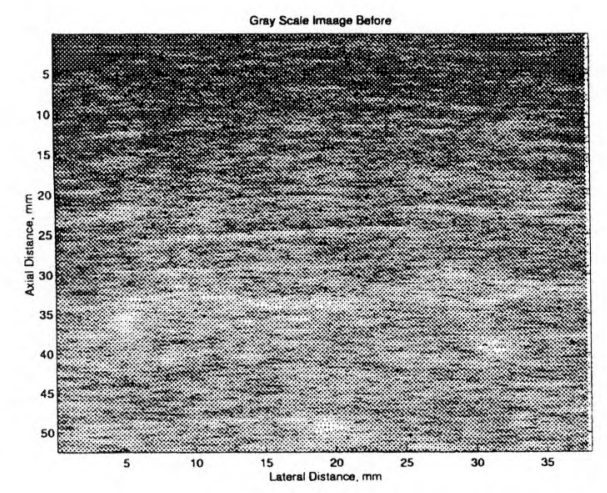

(a)

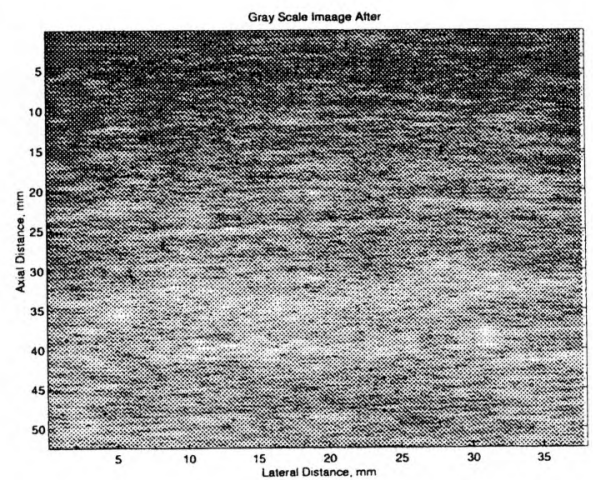

(b)

Figure 3. B-scan gray scale images of the sample before (a), and after (b) the lesion formation.

As seen from Section 3.1.2, one can extract the attenuation coefficient from the center frequency though curve fitting algorithm. Since it is known that attenuation changes dramatically with changes in the tissue state due to thermal coagulation, it is expected that extracting this parameter should be a good indicator of the change in tissue state. Figure 4 is a subtraction image of the center frequency images obtained from the before and after images shown in Figure 3. Significant change in the center frequency can be seen in a region extending from $35-45 \mathrm{~mm}$ in the range direction and $13-26 \mathrm{~mm}$ in the lateral direction. To the best of our knowledge, this is the first such image obtained in reflection mode for this type of application. This result strongly suggest that it will be possible to obtain quantitative images from beamformed RF data that correlate with the damage to the tissue.

\section{SELF-GUIDED PHASED ARRAY SYSTEM FOR NONINVASIVE SURGERY}

The system described in Figure 1 provides an example of image-guided therapeutic phased array. In that case, the image guidance is provided by an independent diagnostic imaging system. Other implementations of image-guided phased arrays with ultrasound, ${ }^{16} \mathrm{MRI},{ }^{15}$ and $\mathrm{CT}^{19}$ have been proposed. In all of these implementations, some form of transformation from the imaging coordinate system to the therapeutic array coordinate system is needed. This may be accomplished with 


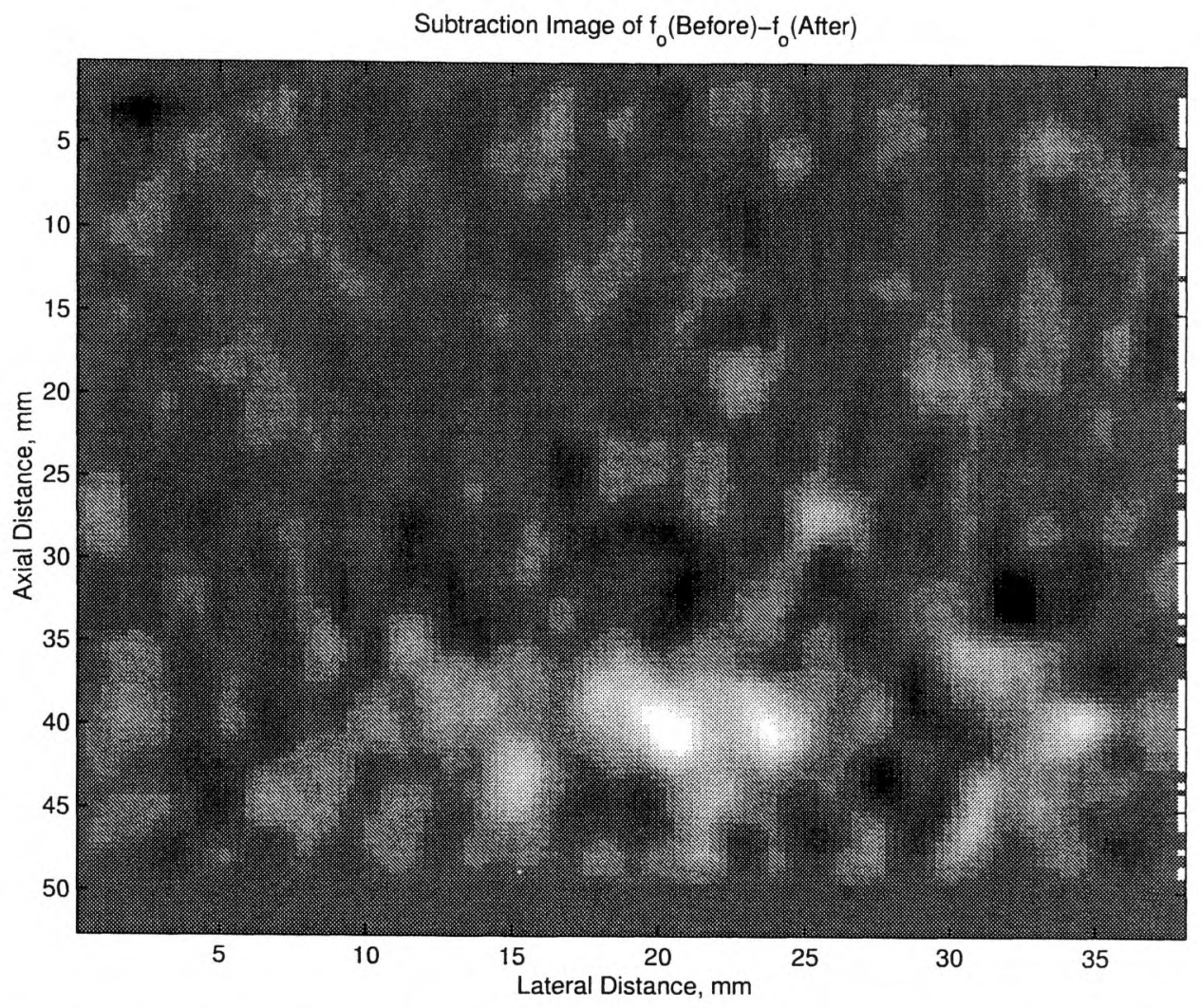

Figure 4. A subtraction image of the central frequency values obtained from the before and after images shown in Figure 3.

various degrees of difficulty depending on the specific imaging modality and imaging geometry.

An attractive alternative for the above paradigm is the use of the same therapeutic applicator to perform imaging. This was facilitated by recent advances in piezocomposite transducer technology. The main advantage of this proposed approach is the use of of the same coordinate system for both therapy and imaging. Therefore, refocusing information obtained for improving image quality are directly applicable to improving the heat localization to the target. Furthermore, acoustic obstacles and critical structures can be directly identified and methods for incorporating them in optimization of focusing are easily obtainable using imaging techniques and are directly applicable to the therapeutic array.

In this section, we describe our recent efforts on developing such phased array systems and appropriate algorithms for their use as imaging transducers. 
(a)

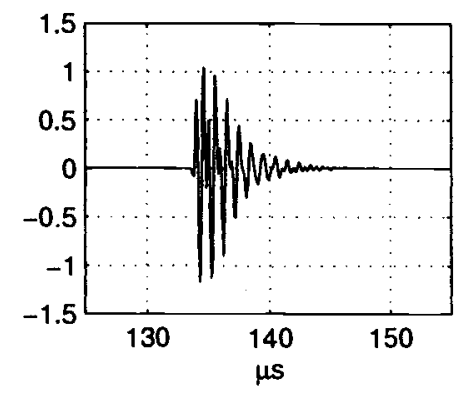

(c)

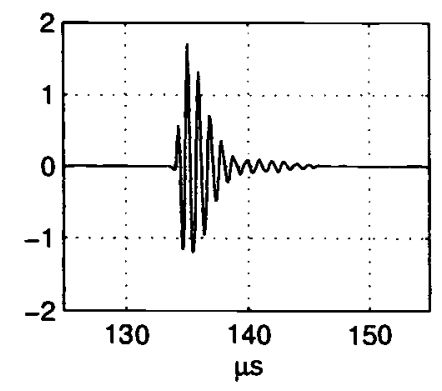

(b)

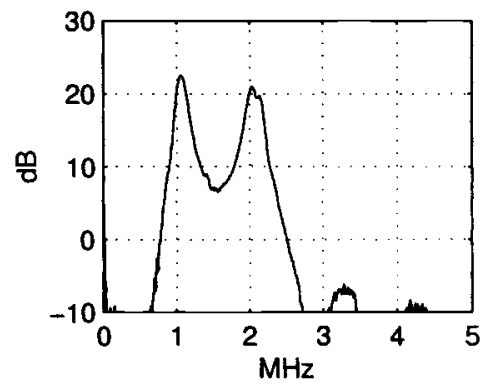

(d)

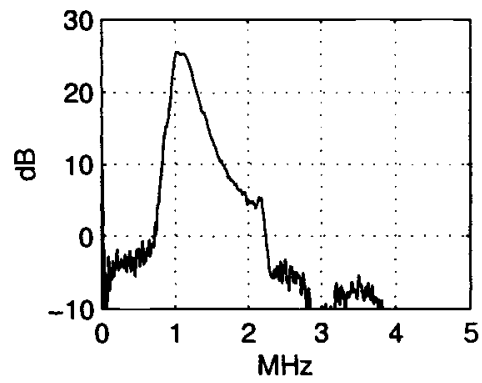

Figure 5. Pulse-echo response measured from a flat reflecting surface. A center element was pulsed and a neighbor element was used in receive. (a) and (b) Time and frequency domain representations when no matching circuit was used. (c) and (d) Time and frequency domain representations when a series low-Q inductor was used to select the $1.1 \mathrm{MHz}$ resonant mode and to reduce the ring down response.

\subsection{Transducer}

A spherical-shape piezocomposite 64-element 1-D array therapeutic transducer (Imasonic, France) operating at $1.1 \mathrm{MHz}$ was used. The echo response received by one of the central elements, when pulsing a neighbor element (Panametrics Pulser Receiver 5052-PR), a thick flat Plexiglas reflector being at the focal distance, is given in Fig. 5a. The corresponding frequency response (Fig. 5b) indicates that the transducer has two predominant resonance frequencies, at 1.1 and $2.1 \mathrm{MHz}$, respectively. When the transducer operates in therapeutic mode, a high- $Q$ resonant matching circuit is used to drive the individual transducer elements at $1.1 \mathrm{MHz}$. When the transducer operates in imaging mode, low-Q series inductors were used to select the $1.1 \mathrm{MHz}$ operating frequency, providing an echo with relatively short duration (Fig. 5c). The -6dB full width bandwidth of the pulse-echo system in this case was measured to be $37 \%$, the frequency response being shown in Fig. $5 \mathrm{~d}$. 


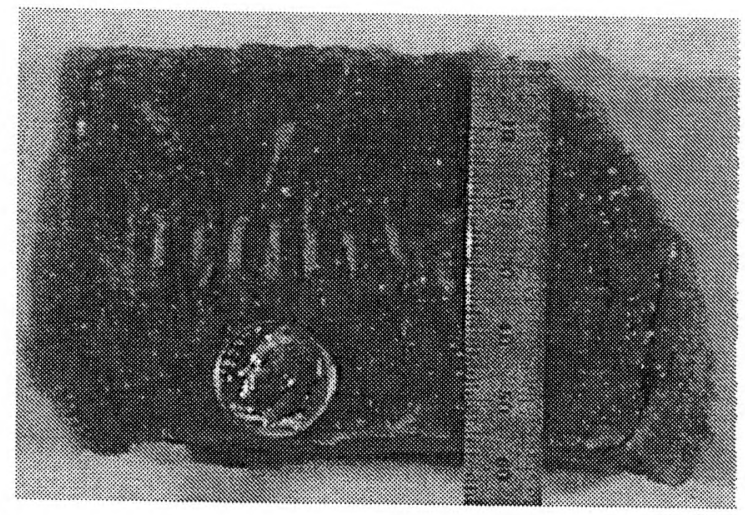

Figure 6. Lesions formed in beef muscle during an in vitro HIFU experiment.

\subsection{Therapeutic Characterization}

A custom designed phased array driving system was used to independently drive each of the 64 elements of the array with control of amplitude and phase. Series inductors are used to obtain a high-Q resonant peak at the operating frequency. The output acoustic power, when driving the transducer with the current system, can be varied from 0 to $510 \mathrm{~W}$, depending on the amplitude of the sinusoids used to drive the elements. The acoustic output power was measured in water using a force balance approach while focusing the transducer at its geometric center (all elements driven in phase).

In order to demonstrate the therapeutic capability of this system, a series of lesions were created in an in vitro beef muscle HIFU experiment. ${ }^{6}$ Eleven single focus sonications were delivered $30 \mathrm{~mm}$ deep within the tissue, and laterally spaced by $5 \mathrm{~mm}$, being distributed from $-25 \mathrm{~mm}$ to $+25 \mathrm{~mm}$ from the geometric center of the transducer. The total acoustic power was fixed at $200 \mathrm{~W}$ and the duration of each sonication was linearly varied from $0.25 \mathrm{sec}$ to $2.75 \mathrm{sec}$. The tissue was allowed to cool down for a sufficiently long time ( 3 minutes) between successive sonications, therefore avoiding temperature build up between sonications. The tissue was cut along the scanned plane and lesions were observed for all sonications with duration longer than $1.25 \mathrm{sec}$, as shown in Fig. 6.

\subsection{Pulse-Echo Imaging}

\subsubsection{Data collection system}

Imaging data was obtained by driving the transducer in pulse-echo mode. The transmitted pulses were generated by a pulser/receiver (Panametrics 5052-PR). The received echoes were first amplified by $30 \mathrm{~dB}$ using an ultrasound signal amplifier (NTR pfs-005a). The transmit and receive circuitry were connected to the array elements through a VXI programmable $64 \times 4$ relay matrix (Tektronix VX4380). Two different data acquisition systems were used to digitize the data (Fig. 7). 
474 / Critical Reviews Vol. CR75

The first digitizer consists of a digital signal analyzer (Tektronix DSA-601), the captured data being transfered to the controlling workstation through a GPIB interface. The duration of the data collection in this case is limited by the speed of the digitizer, a complete set of $64 \times 64$ A-lines taking about 3 hours.

In order to reduce the total duration of the data collection, a gated digitizer was implemented. The data is digitized using a Burr Brown ADS-801 12-bit fastA/D operated at $16 \mathrm{MHz}$ (data sampling frequency). Gating is implemented using conventional fast TTL logic. The digitized data is stored in a dual port $128 \mathrm{Mb}$ memory buffer, and transfered at slower rate to the controlling workstation. The duration of the acquisition is limited in this case by the dead time of the relay matrix after each switching (approximately $40 \mathrm{~ms}$ ), a complete set of $64 \times 64 \mathrm{~A}$-lines being acquired in about 3 minutes. The current prototype implementation of this circuit provides data with lower SNR than the data obtained using the Tektronix digital signal analyzer. This increases the level of the background noise in the reconstructed image, ultimately limiting the image dynamic range. Nevertheless, the system is still capable of providing good image quality, as seen in Fig. 12.

\subsubsection{Synthetic aperture imaging}

Images were obtained using a full synthetic aperture technique. ${ }^{31}$ In this case the system is in focus at every pixel of the image, in transmit and receive. Furthermore, in order to reduce side-lobes, apodization of the transmit and receive apertures was applied by weighting the echo responses with a Kaiser window ${ }^{34}(\beta=3.25)$. The image pixel at coordinates $\left(x_{p}, z_{p}\right)$ was therefore computed by (Fig. 8):

$$
I\left(x_{p}, z_{p}\right)=\sum_{i=1}^{64} \sum_{j=1}^{64} A_{i} \cdot B_{j} \cdot s_{i, j}\left[\left(R_{i p}+R_{j p}\right) / c\right],
$$

where $i$ is the transmit element index, $j$ is the receive element index, $A_{i}$ is the transmit apodization weight at element $i, B_{j}$ is the receive apodization weight at element $j, R_{i p}$ and $R_{j p}$ are the distances from the transmit and receive elements, respectively, from the image pixel, $c$ is the speed of sound in the medium being imaged, and $s_{i, j}(t)$ is the base-band converted echo acquired when transmitting with element $i$ and receiving with element $j$. The images were first sampled in the $R \times \sin (\theta)$ domain, and then scan converted into a Cartesian spatial sampling.

\subsection{Results}

\subsubsection{Multiple-wire phantom}

A multiple-wire phantom consisting of thirteen nylon wires spaced by $10 \mathrm{~mm}$ along a horizontal line was used to characterize the point spread function of the imaging system described above (Fig. 9). The phantom was aligned so that the middle wire was located at the geometric center of the spherically-shaped therapeutic transducer. The phantom and transducer were immersed in water to provide acoustic coupling. A full $64 \times 64$ data-set was collected using the setup represented in Fig. 7, the data being digitized by the Tektronix digital signal analyzer. 


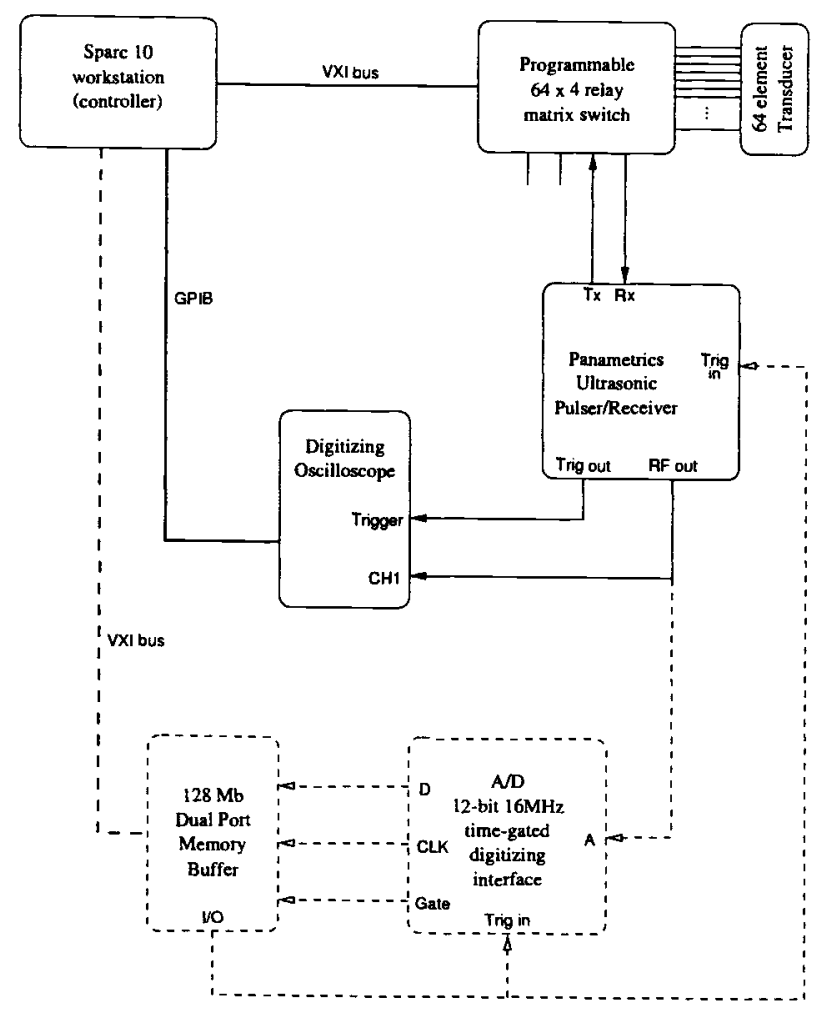

Figure 7. Schematics of the circuitry used in the imaging experiments. When the custom made gated digitizer is used, the digital oscilloscope is replaced by the blocks in dashed lines.

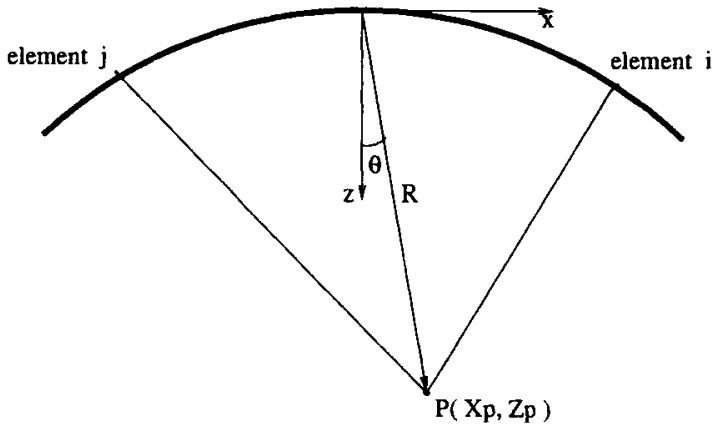

Figure 8. Coordinate system used in the synthetic aperture imaging system. 
476 / Critical Reviews Vol. CR75

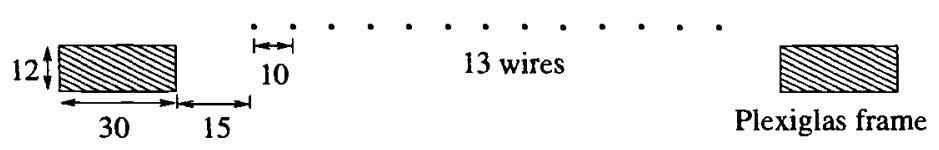

Figure 9. Cross section of the multiple wire phantom. The 13 wires are indicated as well as the Plexiglas frame that holds the wires. Dimensions are indicated in millimeters.

The pulse-echo synthetic aperture reconstructed image is shown in Fig. 10 at dynamic ranges of $20,30,40$, and $50 \mathrm{~dB}$, where all images are referenced to the peak value in the reconstructed region. At $20 \mathrm{~dB}$ seven central wires are seen. As this transducer is highly focused, and no compensation for element directivity was applied during reconstruction, objects closer to the geometric center of the transducer appear to be brighter than objects farther away. Furthermore, as the aperture is undersampled grating lobes become visible when imaging off-axis (image at 40 and $50 \mathrm{~dB}$ ). At $50 \mathrm{~dB}$ eleven of the thirteen wires are seen.

The results in Fig. 10 indicate that this system provides good image reconstruction of objects close to the transducer geometric center. However, the quality of the image degrades as objects off-center are considered. Although this image degradation is certainly not appropriate for diagnostic systems, in the case of therapeutic guidance the most relevant information is contained in the region undergoing treatment. Typically, this corresponds to the region close to the center of the therapeutic transducer, where the image quality is better.

\subsubsection{Speckle-generating phantom}

In order to characterize the performance of this system with speckle media, a tissuemimicking phantom was designed, its dimensions being given in Fig. 11. The phantom is made of rubber material (plastic for making plastic worms, M-F Manufacturing Co., Fort Worth, TX), with embedded polystyrene scatterers (Amberlite IR-120plus, Sigma Chemical Co., St. Louis, MO). The scatterers are homogeneously distributed within the phantom, except for a $12.7 \mathrm{~mm}$ diameter cyst region, that was filled with water. The phantom and the transducer were immersed in water to provide acoustic coupling. The phantom was aligned so that the cyst region corresponded to the geometric center of the therapeutic transducer, as indicated in Fig. 11. A full $64 \times 64$ data set was collected using the experimental setup shown in Fig. 7, the custom gated digitizer being used in this case.

The synthetic aperture image of the phantom is shown at four different dynamic ranges, namely $20,30,40$ and $50 \mathrm{~dB}$, referenced to the peak value detected within the imaged region. The image shows the cyst region, illustrating the performance of the system in the vicinity of the focal region. The strong reflection at the top and bottom edges of the cyst are caused by the acoustic impedance mismatch between the rubber material and water. 


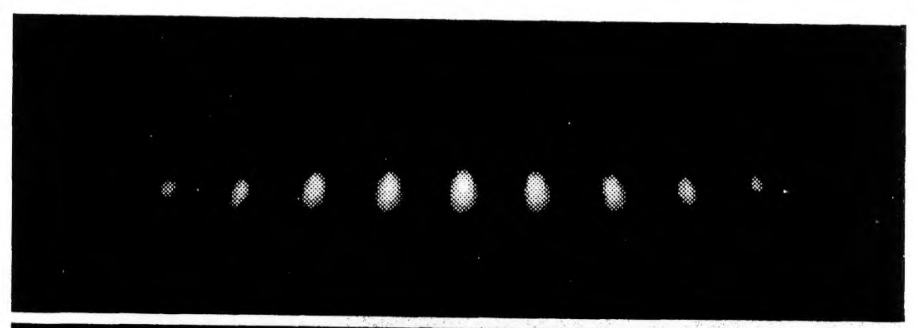

$20 \mathrm{~dB}$

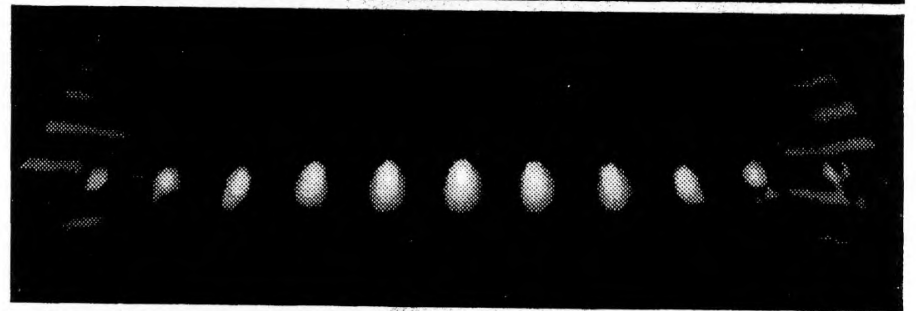

$30 \mathrm{~dB}$

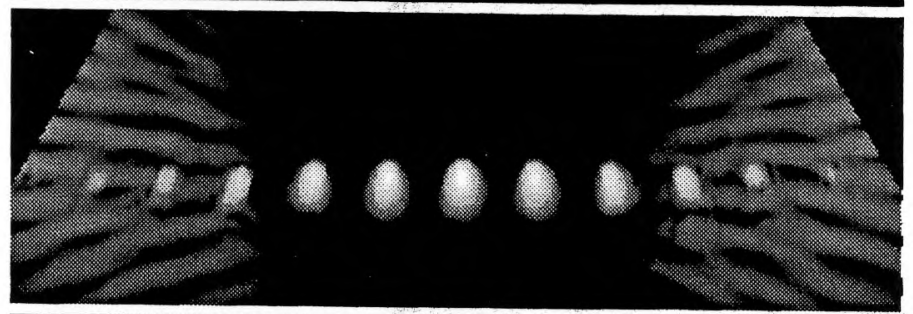

$40 \mathrm{~dB}$

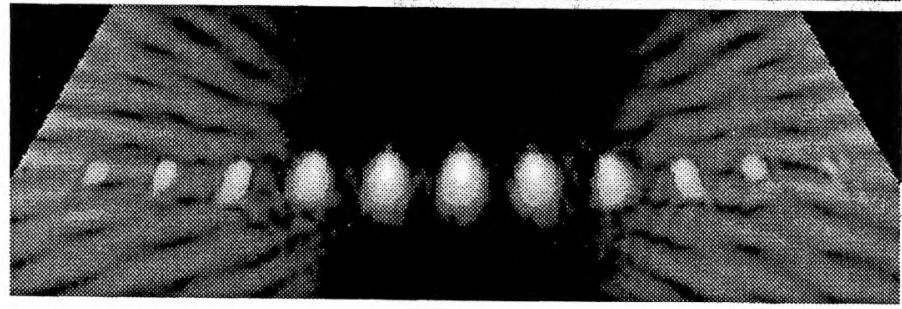

$50 \mathrm{~dB}$

Figure 10. Image of the multiple wire phantom shown at different dynamic ranges. The vertical direction corresponds to axial depth in the interval from $80 \mathrm{~mm}$ to $120 \mathrm{~mm}$. The horizontal direction corresponds to lateral from $-60 \mathrm{~mm}$ to $+60 \mathrm{~mm}$. 


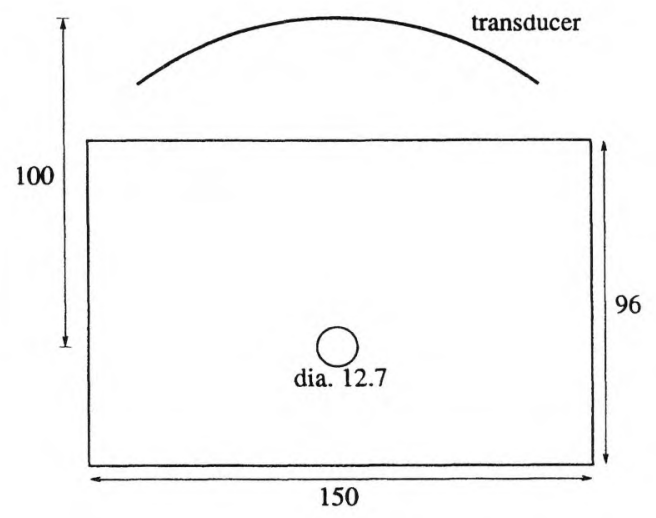

Figure 11. Dimensions of the speckle generating phantom and its position relative to the transducer. Dimensions are indicated in millimeters.

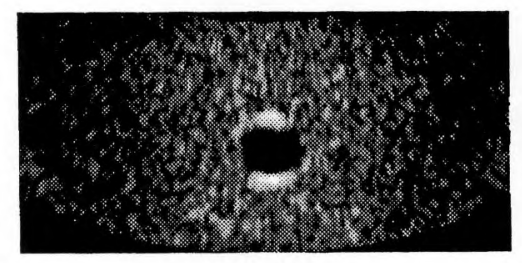

$30 \mathrm{~dB}$

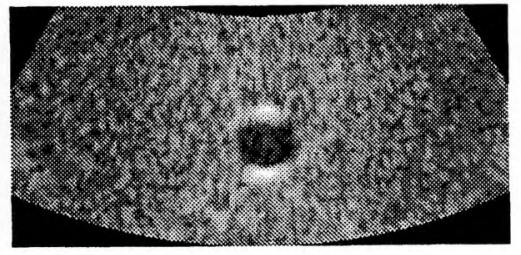

$50 \mathrm{~dB}$

Figure 12. Image of the speckle generating phantom shown at different dynamic ranges. The vertical direction corresponds to axial depth in the interval from $70 \mathrm{~mm}$ to $120 \mathrm{~mm}$. The horizontal direction corresponds to lateral from $-50 \mathrm{~mm}$ to $+50 \mathrm{~mm}$. 
Matching the Energy Source to the Clinical Need / 479

\section{DISCUSSION}

\subsection{Temperature Imaging}

The algorithm described in this paper was developed based on an approximate theoretical model and was verified in tissue mimicking phantoms under conditions similar to those assumed in this paper. We have also performed many experiments in tissue media that have conclusively shown that the time shifts produced even by small to moderate temperature rise are measurable and correspond to expected spatiotemporal distribution of temperatures due to localized heating sources. However, there are several limitations of this temperature imaging algorithm that require further development before this method finds clinical application in in-vivo clinical and animal testing experiments. In the following, we enumerare these limitations and describe efforts to overcome them. Citations of relevant work in this regards are given where appropriate.

1. Motion and Deformation of Heated Tissue: This is probably the most serious limitation of the temperature estimation algorithm as it is presented in this paper. Tissue motion and deformation can lead to echo shifts that are 2 3 orders of magnitudes higher than the echo shifts produced by temperature changes. Left uncorrected, the echo shifts due to tissue motion and deformation can mask those shifts produced by temperature changes. We have proposed $^{12}$ a two-step approach that compensates for uniform motion and deformation in performaing the temperature estimation problem. A modified version of this algorithm will be needed to correct for nonuniform motion and deformation as can be seen in liver and other soft organs. This work is currently underway.

2. Inhomogeneity of the Temperature Coefficients: Knowledge of the temperature coefficients of the speed of sound and the thermal expansion are needed for obtaining a quantitative measure of temperature change without the need for calibrating temperature measurements (e.g., through thermocouple measurements.) At this point, we rely on (reasonable) estimates of these parameters from the literature and from off-line measurements. This is likely to continue to be the case due to the lack of accurate in-situ measurements and to the variability of these parameters. Fortunately, however, the use of approximate values of these parameters produces only a small error in temperature estimation. This has been confirmed in both phantom and tissue heating experiments with refrence temperatures measured using calibrating thermocouples.

3. Nonuniform Baseline Temperature: The algorithm shoun in this paper assumes a uniform baseline temperature. This only correct at the begining of heating, but is not valid for subsequent frames after temperaure buildup has occured. This may be a real limitation if one attempts to image temperatures in the coagulative necrosis range. In the hyperthermia and image guidance range, however, the nonuniformity of the baseline temperature is not a major limitation. A $10^{\circ} \mathrm{C}$ change in tissue temperature produces a $7 \%$ change in the 
480 / Critical Reviews Vol. CR75

proportionality factor in the temperature estimation equation. In practice, we have used the algorithm described above in tracking temperature changes up to $15^{\circ} \mathrm{C}$ successfully. Methods for extending the current algorithm beyond this range are being developed.

\subsection{Damage Assessment}

The result shown in Figure 4 clearly demonstrate that the center frequency is very sensitive to changes in the tissue state after thermal coagulative necrosis. However, this image is still not of sufficient quality to provide easy visual characterization of thissue damage. At this point, it appears very likely that the center frequency will be one of several parameters extracted and processed (on a statistical basis) to characterize the state of the tissue. However, the importance of this result cannot be overemphasized. While MRI and X-ray CT are ahead of ultrasound in terms of quantitative damage assessment, ultrasound is superior in terms of portability, realtime imaging capability, cost, and complexity. If this and many of our in-vitro results can be demonstrated in in-vivo tissues, then a major limitation towards ultrasound guided noninvasive surgery will be removed. Given other advances in noninvasive temperature imaging ${ }^{11}$ and dual phased arrays for imaging and therapy, ${ }^{13}$ it is now possible to envision a self-guided ultrasound phased array that is capable of delivering the HIFU pulses, monitor the progression of the treatment, and perform a post treatment evaluation. This can save time and resources

We should note that the image shown in this paper is a subtraction image. While subtraction images are very useful and help identify before and after type changes as we have in this experiment, they are not always possible. In practice, high temperature procedures cause tissue deformations. These, in addition to tissue motion during the procedure, make it very hard if not impossible to produce such subtraction images reliably. It is therefore necessary to develop practical algorithms for motion compensation. In addition, image registration techniques with deformable models will be needed. We are currently investigating such tools. It is hoped that more research effort will be directed to this area in the near future.

\subsection{Self-Guided Ultrasound Phased Arrays for Noninvasive Surgery}

Poor acoustic penetration in tissue usually limits the applicability of synthetic aperture imaging techniques. ${ }^{31}$ However, for the system presented in this paper the transducer elements are designed to have very high efficiency in transmit. Furthermore, the received echo signal is relatively strong due to the element large surface area as well as its focusing along the elevation direction. Moreover, in the case of diagnostic systems, the maximum acoustic intensity is limited to a relatively low value, in order to avoid potential for damage or risk to the patient. In a highintensity focused ultrasound therapeutic context, however, the acoustic intensities used in the treatment are much higher than those generated by diagnostic systems. Therefore, when operating the therapeutic transducer in imaging mode to provide 
guidance for therapeutic procedures, as suggested in this paper, it is potentially acceptable to use acoustic intensities that exceed the limits specified for conventional diagnostic systems.

Motion of the object being imaged also imposes practical limitations to the applicability of synthetic aperture imaging techniques. In the case of the system presented in this paper, it should be possible to acquire a full $64 \times 64$ data set within $12 \mathrm{~ms}$, assuming that the echoes received at the 64 elements are collected simultaneously, and a maximum imaged depth of $150 \mathrm{~mm}$ is used. With the exception of cardiac applications, one can assume that most tissues will not move faster than $10 \mathrm{~mm} / \mathrm{s}$. In this case, the maximum error due to motion within the collection of a single frame $(12 \mathrm{~ms}$ ) will be limited to $0.16 \mu \mathrm{s}$, or $\lambda / 6$ ( $\lambda=$ wavelength) for an imaging frequency of $1 \mathrm{MHz}$.

\section{CONCLUSIONS}

We have addressed three aspects of the use of ultrasound phased array technology in noninvasive thermal surgery. These are temperature imaging, quantitative imaging for damage assessment, and dual-mode self-guided array systems. The latter is unique to ultrasound and could prove to be key to the overall development of noninvasive surgery. However, at the very least, this approach may be the only viable approach for noninvasive surgery with ultrasound arrays. Given the complexity of ultrasound wave propagation, the issue of guiding ultrasound with a separate imaging modality may prove to be quite difficult. Self-guided phased arrays will be the only viable alternative. Much more research is needed in this area, but the early attempts are quite promising. With the availability of high-power arrays with improved bandwidths over what we presented in this paper, we can only expect significant improvements in image quality.

Temperature imaging with ultrasound is also at an early development stage and will require significant effort before it can be used reliably in clinical situations. However, even at this early stage, one can argue that ultrasound stands the best chance of being used for localizing the heat source in multidimensional spatio-temporal maps in realtime and with very high spatial resolution. The main challenge at this point is the development of quantitative calibration-free temperature imaging algorithms. We predict that semiquantitative and calibration-aided quantitative temperature imaging with ultrasound will become available within the next few years, especially with the introduction of $3 \mathrm{D}$ imaging systems by several manufacturerers. This feature will eliminate one of the major limitations of ultrasound temperature imaging. In particular, out of plane motion will be easily tracked and, therefore, speckle tracking for temperature estimation will improve in robustness and accuracy. This is likely to prove to be a very fruitful research area for ultrasound imaging in general, but for temperature imaging in particular.

In the area of damage assessment, ultrasound currently stands at a relative disadvantage. The results shown in this paper are representative of how little was achieved in this area. It is fair to say that no serious attempt at developing ultrasound imaging tools for damage assessment has been taken. Similar to what we have 
shown in this paper, several researchers have shown before and after type images of certain tissue parameters that reflect changes due to thermal lesion formation. However, these results are still not quantitative and, to the best of our knowledge, no specific imaging algorithm for damage characterization has been proposed in the literature. However, ultrasound wave propagation in tissue media is fundamentally quantitative and is sensitive to tissue structure as well as scattering characteristics. Ultrasound continues to hold significant promise in this regard. Several recent developments in harmonic imaging, elastography, and vibroacoustic emission imaging appear to offer the most promise for damage characterization. At the time of writing this article, some preliminary work has been presented in these areas. More work is needed to corroborate these results and establish repeatibility.

In summary, the promise of ultrasound phased array technology in the area of noninvasive surgery has never looked better. However, much more work is needed to bring this promise to fruitful clinical application. We note that the interest in therapeutic ultrasound is quite high in both industry and at academic institutions. It is hoped that the collective effort of this community will move this research forward and bring us closer to true noninvasive surgery early in the new millenium.

\section{Acknowledgements}

This work was funded by Grant CA 66602 from the National Institutes of Health. Many thanks to former students P. VanBaren, C. Simon, and K. Oweiss for help with the data collection and storage.

\section{REFERENCES}

1. R.Seip, P.VanBaren, C.Simon, and E.Ebbini, "Non-invasive spatio-temporal temperature change estimation using diagnostic ultrasound," IEEE Ultrason. Symp. Proc., pp.1613-1616, Nov. 1995.

2. P. VanBaren and E. Ebbini, "Multipoint temperature control during hyperthermia treatments: Theory and simulations," IEEE TRans. BME, vol. 42, no. 8, pp. 818-827, Aug. 1995.

3. R. Seip, P. VanBaren, C. Cain and E. Ebbini, "Noninvasive real-time multipoint temperature control for ultrasound phased array treatments," IEEE Trans. UFFC, vol. 43, no. 6, pp. 1063-1073, Nov. 1996.

4. T. Sheljaskov, R. Lerch, M. Bechtold, K. Newerla, and U. Schätzle, "A phased array antenna for simultaneous HIFU therapy and sonography," IEEE Ultrason. Symp. Proc., pp.1527-1530, Nov. 1996.

5. T. Sheljaskov, R. Lerch, A. Fuchs, and U. Schätzle, "A phased array antenna for simultaneous thermotherapy and sonography," IEEE Ultrason. Symp. Proc., Oct 1997.

6. P. VanBaren, C. Simon, R. Seip, T. Solf, C. Cain, and E. Ebbini, "Imageguided phased array system for ultrasound thermotherapy," IEEE Ultrason. Symp., pp. 1269-1272, Nov. 1996. 
7. T. Loupas, J. Powers, and R. Gill, "An axial velocity estimator for ultrasound blood flow imaging, based on a full evaluation of the Doppler equation by means of a two-dimensional autocorrelation approach," IEEE Trans. UFFC, vol. 42, no. 4, pp. 672-688, July 1995 .

8. J. Greenleaf, "Computerized transmission tomography," in Methods of Experimental Physics, vol. 19 Ultrasonics, Academic Press, London, 1981.

9. R. Nasoni, T. Bowen, W. Connor, R. Sholes, "In vivo temperature dependence of ultrasound speed in tissue and its application to noninvasive temperature monitoring," Ultrasonic Imaging, vol. 1, no. 1, pp. 34-43, 1979.

10. C. Simon Two-Dimensional Temperature Estimation Ph.D. Dissertation, The University of Michigan, Ann Arbor, 1998.

11. C. Simon, P. VanBaren, and E. Ebbini, "Two-dimensional temperature estimation for ultrasound thermotherapy using diagnostic ultrasound," IEEE Trans. Ultrason., Ferroelec., Freq. Contr., vol. 45, no 4, pp. 1088-1099, July 1988.

12. C. Simon, P. VanBaren, and E. Ebbini, "Motion compensation algorithm for non-invasive two-dimensional temperature estimation using diagnostic pulseecho ultrasound," SPIE Bios: Surgical Applications of Energy, January 1998.

13. C. Simon, J. Shen, T. Hall, and E. Ebbini, "Combined ultrasound image guidance and therapy using a therapeutic phased array," SPIE: Medical Imaging 1998, vol. 3341, pp. San Diego, Feb. 1998.

14. E. Ebbini and C. Simon, "Combined imaging and therapy with piezocomposite phased arrays," 1998 IEEE Ultrasonics Symposium, vol. 2, pp. 1555-1558, 1998.

15. K.Hynynen, "Review of ultrasound therapy," 1997 IEEE Ultrasonics Symposium, vol. 2, pp. 1305-1313, 1997.

16. N. Sanghvi et al., "Noninvasive surgery of prostate tissue by high-intensity focused ultrasound," IEEE Trans. UFFC, vol. 43, no. 6, pp. 1099-1110, Nov. 1996.

17. R. Seip, Feedback for ultrasound thermotherapy, Ph.D. Dissertation, The University of Michigan, 1996.

18. E. Ebbini, P. VanBaren, and C. Simon, "Image-guided noninvasive surgery with ultrasound phased arrays," SPIE Bios: Surgical Applications of Energy, 1998.

19. J. Jenne, M. Bahner, J. Spoo, P. Huber, R. Rastert, I. Simiantonakis, W. Lorenz, and J. Debus, "CT on-line monitoring of HIFU therapy," IEEE Ultrason. Symp., 1997.

20. R. Seip and E. Ebbini, "Non-invasive estimation of tissue temperature response to heating fields using diagnostic ultrasound," IEEE Trans. Biomed. Eng., vol. 42, no.8, pp. 828-839, Aug. 1995.

21. R. Moreno, C. Damianou, and N. Sanghvi, "Tissue temperature estimation in-vivo with pulse-echo," IEEE Ultrason. Symp., pp. 1225-1229, Nov. 1995.

22. K.Paulsen, M.Moskowitz, T.Ryan, S.Mitchell, and P.Hoopes, "Initial in vivo experience with EIT as a thermal estimator during hyperthermia," Int. J. Hyperthermia, vol. 12, no. 5, pp. 573-591, Sept. 1996. 
484 / Critical Reviews Vol. CR75

23. P.Meaney, K.Paulsen, A.Hartov, and R.Crane, "Microwave imaging for tissue assessment: Initial evaluation in multitarget tissue-equivalent phantoms," IEEE Trans. Biomed Eng., vol. 43, no. 9, pp. 878-890, Sept. 1996.

24. B. Fallone, P. Moran, and E. Podgorsak, "Noninvasive thermometry with a clinical X-ray scanner," Med. Phys., vol. 9, no. 5, pp. 715-721, 1982.

25. D. Parker, "Applications of NMR imaging in hyperthermia: An evaluation of the potential for localized tissue heating and noninvasive temperature monitoring," IEEE Trans. Biomed. Eng., vol. 31, no. 1, pp. 161-167, 1984.

26. J. Poorter, C. Wagter, Y. Deene, C. Thomsen, F. Stahlberg, and E. Achten, "Noninvasive MRI thermometry with the proton resonance frequency (PRF) method: In vivo results in human muscle," Magn. Reson. Med., vol. 33, pp. 74-81, 1995.

27. W. Straube and R. Arthur, "Theoretical estimation of the temperature dependence of backscattered ultrasonic power for noninvasive thermometry," Ultrasound in Med. \& Biol., vol. 20, no. 9, pp. 915-922, 1994.

28. S. Ueno, M. Hashimoto, H. Fukukita, and T. Yano, "Ultrasound thermometry in hyperthermia," in IEEE Ultrason. Symp., pp. 1645-1652, 1990.

29. A. Malcom, and G. ter Haar, "Ablation of tissue volumes using high intensity focused ultrasound," Ultrasound in Med. and Biol., vol22, pp. 659-699, 1996.

30. C. Simon, P. VanBaren, and E. Ebbini, "Quantitative analysis and applications of non-invasive temperature estimation using diagnostic ultrasound," in 1997 IEEE Ultrasonics Symposium, pp. 1319-1322, October 1997.

31. K. Thomenius, "Evolution of ultrasound beamformers," IEEE Ultrason. Symp., pp. 1615-1622, Nov. 1996.

32. I. Hein, and W. O'Brien Jr., "Current time-domain methods for assessing tissue motion by analysis from reflected ultrasound echoes - a review," IEEE Trans. Ultrason., Ferroelec., Freq. Contr., vol. 40, no. 2, pp. 84-102, March 1993.

33. R. Nasoni and T. Bowen, "Ultrasonic speed as a parameter for non-invasive thermometry," in Non-invasive temperature measurement, S. Mizushina, editor, volume 1, pp. 95-107, Gordon and Breach Science Publishers, 1989.

34. A. Oppenheim, and R. Schafer, Discrete-Time Signal Processing, Prentice-Hall, Englewood Cliffs, 1989.95-107, Gordon and Breach Science Publishers, 1989. 